\title{
Lockin thermography as a measurement technique in heat transfer
}

\author{
M. Wandelt and W. Roetzel
}

\author{
Universităt der Bundeswehr Hamburg, Institut for Thermodynamik, D-22039 Hamburg, Germany
}

\begin{abstract}
An experimental temperature oscillation technique is described for determining local distributions of the heat transfer coefficient or local distributions of the thermal diffusivity of heat transferring walls. By heating uniformly one surface of the wall with sinusoidally modulated energy a temperature oscillation is generated which results in a wavelike propagation behavior of heat flow and temperature within the wall. The characteristic of the temperature oscillations at both faces of the wall depends directly on the local heat transfer conditions and the thermal diffusivity of the wall material. So the local values of the heat transfer coefficient or the thermal diffusivity can be calculated from the measured amplitudes or from the phases of the temperature waves at the surfaces. To demonstrate the applicability of the method first experiments were performed. The measured results agree reasonably well with data obtained from literature.
\end{abstract}

\section{Introduction}

Because of their simplicity and accuracy temperature oscillation techniques obtained an increasing importance for measuring purposes in heat transfer. The basic idea of these techniques is to supply modulated energy to the testing object which results in a wavelike propagation behavior of heat flow and temperature within the object material. By measuring and analyzing the temporal and spatial propagation behavior of the thermal waves, numerous thermophysical parameters of the testing object can be determined.

With an earlier developed method [1] for measuring the local heat transfer coefficient or the local thermal diffusivity periodic temperature oscillations are optically generated at one spot of the object surface by periodic heating with a focused laser beam. This method was then refined by Wandelt [2] to achieve a much higher accuracy. Measurements which cover the whole object surface have to be performed point by point in a rasterlike fashion. At each point one has to wait a minimum time, until the local stationary state is achieved. So measuring times can be long which might be not acceptable for practical applications.

The technique presented overcomes the restrictions of the method described above. Local distributions of the heat transfer coefficient as well as local distributions of the thermal diffusivity can be determined for the whole testing object within a short time.

\section{Measurement principle}

The measurement principle is illustrated in figure 1. Sinusoidally modulated thermal energy is supplied uniformly to the whole surface of the object under consideration. From the surface a nearly plane thermal wave propagates into the material after the initial transient behavior. The local amplitudes and phases of the temperature waves at both sides of the wall depend on the properties of the wall, the frequency of the oscillation and the heat flux to the surrounding.

By measuring the local temperature oscillations at each surface point and by evaluating the local phases and amplitudes of these oscillations, a phase angle image and an amplitude image can be extracted for the whole surface. In principle, a map of the local heat transfer coefficient or a map of the locally varying thermal diffusivity can now be calculated from the data of both the images.

For monitoring and recording thermal waves a rapid infrared scanning device (AGEMA THV900LW/ST) is used, which allows a non-contacting measurement of the small wall temperałure changes with high thermal, spatial and time resolution. The thermal information from all scanned points are analyzed with a PC. 


\section{Analysis}

The relation between the temperature oscillations at both sides of the wall, the thermophysical properties of the wall material and the heat transfer to the surrounding is described by the energy-equation and the boundary conditions.

Under steady oscillation conditions, the wall temperature can be subdivided into the steady state contribution (local time averaged temperature) and the steady periodic part $\Theta(z, t)$, which represents the actual temperature oscillation. With a few simplifying assumptions the steady periodic part is described by

$$
\begin{aligned}
& \alpha \frac{\partial^{2} \Theta(z, t)}{\partial z^{2}}=\frac{\partial \Theta(z, t)}{\partial t}, \\
& \left(\frac{\partial \Theta(z, t)}{\partial z}\right)_{0}=\frac{h_{0}}{k} \Theta(z=0, t), \\
& \left(\frac{\partial \Theta(z, t)}{\partial z}\right)_{\delta}=\frac{\bar{q}}{k} m \sin \omega t-\frac{h_{\delta}}{k} \Theta(z=\delta, t),
\end{aligned}
$$

where $h_{0}$ and $h_{\delta}$ are the heat transfer coefficients at both sides of the wall and $m$ is the modulation factor of the energy supplied at the heated surface.

A solution of equation (1) with the boundary conditions (2) and (3) can be found on applying the theory of Laplace transforms. For the representation of the solution $\Theta(z, t)$ in the time domain it is useful to introduce three dimensionless groups as new variables. The thickness of the wall and the heat transfer coefficients at the positions $z=0$ and $z=\delta$ are expressed in dimensionless form as

$$
\xi=\delta \sqrt{\frac{\omega}{2 \alpha}}, \quad \psi_{0}=\frac{h_{0}}{\delta \rho c \omega}, \quad \psi_{\delta}=\frac{h_{\delta}}{\delta \rho c \omega} .
$$

In terms of these variables the solution $\Theta(z, t)$ is given as

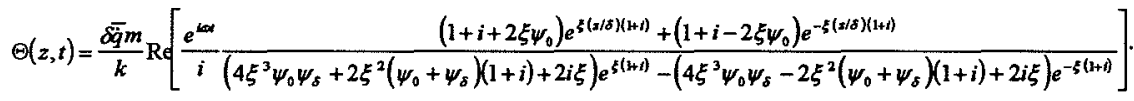

On the other hand the function $\Theta(z, t)$ can also be described by using the local amplitude $U(z)$ and phase $\phi(z)$ of the oscillation which gives the expression

$$
\Theta(z, t)=U(z) \sin (\omega t-\phi(z))=U(z)[\cos \phi(z) \sin \omega t-\sin \phi(z) \cos \omega t] .
$$

By comparing equations (5) and (6) one gets the following formulations for the local amplitudes and phases at $z=0$ and $z=\delta$

$$
\begin{aligned}
& \tan \phi(z=\delta)=\frac{c_{1}+2 \xi \psi_{0} c_{2}+2 \xi^{2} \psi_{0}^{2} c_{3}}{2 \xi \psi_{0}(1+r) c_{0}+2 \xi^{2} \psi_{0}^{2}(1+2 r) c_{1}+4 \xi^{3} \psi_{0}^{3} r c_{2}+c_{3}}, \\
& \tan \phi(z=0)=\frac{2 \xi^{2} \psi_{0}^{2} r(\tan \xi-\tanh \xi)+2 \xi \psi_{0}(1+r) \tan \xi \tanh \xi+\tan \xi+\tanh \xi}{2 \xi^{2} \psi_{0}^{2} r(\tan \xi+\tanh \xi)+2 \xi \psi_{0}(1+r)-\tan \xi+\tanh \xi}, \\
& U^{2}(z=\delta)=\left(\frac{\overline{\delta q} m}{k}\right)^{2} \frac{c_{0}+2 \xi \psi_{0} c_{1}+2 \xi^{2} \psi_{0}^{2} c_{2}}{4 \xi^{4}\left[\psi_{0}(1+r)\right]^{2} c_{0}+8 \xi^{3} \psi_{0}^{3} r(1+r) c_{1}+\left(2 \xi^{2}+8 \xi^{6} \psi_{0}^{4} r^{2}\right) c_{2}+4 \xi^{3} \psi_{0}(1+r) c_{3}}, \\
& U^{2}(z=0)=\left(\frac{1}{k}\right)^{2} \frac{1}{4 \xi^{4}\left[\psi_{0}(1+r)\right]^{2} c_{0}+8 \xi^{5} \psi_{0}^{3} r(1+r) c_{1}+\left(2 \xi^{2}+8 \xi^{6} \psi_{0}^{4} r^{2}\right) c_{2}+4 \xi^{3} \psi_{0}(1+r) c_{3}},
\end{aligned}
$$




\section{http://dx.doi.org/10.21611/qirt.1996.031}

where the following abbreviations are introduced

$$
\begin{array}{ll}
c_{0}=\cosh ^{2} \xi \cos ^{2} \xi+\sinh ^{2} \xi \sin ^{2} \xi, & c_{1}=\cosh \xi \sinh \xi+\cos \xi \sin \xi, \\
c_{2}=\cosh ^{2} \xi \sin ^{2} \xi+\sinh ^{2} \xi \cos ^{2} \xi, & c_{3}=\cosh \xi \sinh \xi-\cos \xi \sin \xi, \\
r=\psi_{\delta} / \psi_{0} . &
\end{array}
$$

As can be seen from equations (7) - (10) the amplitude ratio $U_{0} / U_{\delta}$ and the phases of the temperature oscillations at both sides of the wall depend only on the three variables $\xi, \psi_{0}$ and $r$. So, if two of these parameters are known, the third one can be calculated by using the measured local amplitude ratios and/or the phases at one or both sides of the wall.

\section{Experimental technique and errors}

Theoretically the methods using phase evaluation or amplitude evaluation must give the same results. In the real experiment the measurement errors for the temperature readings have to be taken into account. Depending on the parameter to be measured the sensitivity of the methods to errors of the input parameters $\left(\xi, \psi_{0}\right.$ or $\left.r\right)$ have additionally to be taken into consideration.

\subsection{Evaluation from amplitude ratio}

The ratio $U_{0} / U_{\delta}$ does not depend on the parameter $(\overline{\bar{q}} m) / k$ so that an evaluation of $\psi$ or $\xi$ from measured amplitude data would in principle be possible. Figure 2 shows the ratio of the amplitudes which is plotted in a region of practical interest. One can see, that for the higher values of $\xi$ there is a high sensitivity for $\psi_{0}$ up to $\psi_{0} \approx 2$. Especially for the small values of the dimensionless heat transfer coefficient the amplitude ratio is additionally fairly insensitive for $\xi$. Therefore this parameter range would be suitable for determining $\psi_{0}$. For determining $\xi$ the dimensionless heat transfer coefficient should be chosen high because of the low sensitivity for $\psi_{0}$ and the good sensitivity for $\xi$ in this parameter range. However, with amplitude evaluation there will arise many difficulties in the experimental setup.

\subsection{Evaluation from the phases at $z=\delta$}

Accurate phase measurements are more easily performed than amplitude measurements. In figure 3 the phase $\phi_{5}$ relative to the initiating modulated thermal energy is plotted as a function of $\xi$ and $\psi_{0}$. As can be seen the $\phi_{5}$-method gives a high sensitivity for $\psi_{0}$ with decreasing values of $\psi_{0}$, but at the same time also the sensitivity for $\xi$ is relatively high. So the range of the low values for the dimensionless heat transfer coefficient seems to be suitable for determining $\Psi_{0}$, if $\xi$ is known with sufficient accuracy. For evaluating $\xi$ the $\phi_{5}$-method seems not to be feasible.

\subsection{Evaluation from the phases at $z=0$}

In figure 4 the phase lag $\phi_{0}$ of the temperature waves relative to the initiating heat flux is plotted as a function of the parameters $\xi$ and $\psi_{0}$. The sensitivity for $\psi_{0}$ at the low values of the dimensionless heat transfer coefficient is good while at the same time the sensitivity for $\xi$ is lower than with the $\phi_{5}-$ method. Additionally the sensitivity for $\xi$ at the high values of $\psi_{0}$ is much better than with phase evaluation at the side $z=\delta$. Therefore the technique of evaluating the phases at $z=0$ should be preferred for determining the heat transfer coefficient as well as for determining $\xi$, if the experimental setup allows temperature measurements at this side.

\subsection{Evaluation of the phase difference between $z=0$ and $z=\delta$}

As can be seen in figure 5 this method is rather insensitive to the dimensionless heat transfer coefficient for a wide range of the parameters $\psi_{0}$ and $\xi$ while the sensitivity for $\xi$ is 
Therefore this method is in principle best suited to gain accurate results for $\xi$ while the possibility to determine the heat transfer coefficient with sufficient accuracy can be excluded.

As a result of the above considerations and of some experimental tests, the methods of evaluating the dimensionless heat transfer coefficients or the $\xi$-values from the measured phases $\phi_{0}$ or $\phi_{\delta}$ turn out to be the most reliable ones.

\section{Evaluation of thermal image data}

For the experiments of this work, a stirling cooled AGEMA 900LW/ST scanner was used. During the experiments a series of frames was scanned over several periods $t_{0}$, with a maximum storage speed of 30 frames/s, each frame containing $272 \times 68$ pixels.

From the stored frame-series, the temperature of each pixel is extracted as a function of time. Figure 6 shows a typical plot of the temperature of two pixels, here indexed with " $a$ " and " $\mathrm{b}$ ". From the complete temperature readings the fundamental oscillations with the amplitudes $U_{a}, U_{b}$ and the phases $\phi_{a}, \phi_{b}$ are mathematically extracted. The results are also plotted in figure 6 as the thick continuous lines. Because a periodically varying quantity (local temperature) with known frequency is measured the analysis can be performed by applying frequency selective techniques. These techniques give accurate results even if the signal to noise ratio is very low. For the determination of $\psi$ or $\xi$ by evaluation of the phases, the phase differences of all points in the image relative to the oscillation of the initiating heating energy are calculated. The result is a phase image for the surface of the testing object. The value of $\psi$ or $\xi$ is then determined using equations (7) or (8). Finally the local heat transfer coefficients $h_{0}$ and $h_{\delta}$ or the local thermal diffusivities $\alpha$ are calculated from $\psi_{0}, \psi_{\delta}$ or $\xi$ by solving equation (4).

\section{Experimental results}

As a first test for the applicability of the method some experiments were performed. In these experiments, the local heat transfer coefficients of an air stream flowing along the surface of a thin copper plate were measured with the $\phi_{0}$-technique at the whole surface of the plate. The experimental setup is shown in figure 7.

The air flow velocity and temperature were measured only for the determination of the Reynolds-number $\mathrm{Re}$ and the properties necessary to determine comparative values of the heat transfer coefficient by well established equations. Here the local laminar heat transfer coefficient $h_{x}^{*}$ at the flow length $\mathrm{l}_{\mathrm{x}}$ was determined using the equation of Pohlhausen [3]

$$
h_{x}^{*}=0,5 * \bar{h}_{x}, \quad \bar{h}_{x}=0,664 \frac{k}{l_{x}} \sqrt{\operatorname{Re}_{x}} \sqrt[3]{\mathrm{Pr}} .
$$

The properties of air were taken from the VDI Heat Atlas [4].

In the experiments a phase map of the surface was calculated from the measured temperature oscillations. Merely from the phase information the distribution of the heat transfer coefficient was determined. As an example the result for a flow velocity of $30 \mathrm{~m} / \mathrm{s}$ is given in figure 8 . In this figure a plot of $h_{x}^{*}$ according to equation (12) is added. One can see, that good agreement is obtained between the experimental results and the expected values.

\section{Conclusions}

The results of the simple experimental tests proof and demonstrate that the determination of distributions of heat transfer coefficients by lockin thermography is a convenient and reliable method. Its main advantages are that the measurements for the whole surface of the testing object are performed simultaneously with high spatial resolution and short measuring times. With this method no probing or manipulation at the fluid side are necessary and in most cases the model surface must not be prepared. Often a periodical heat flux of only a few watts will be sufficient to generate the temperature oscillations. Merely the surface temperatures are measured with a non-contacting method. In the experiments of this work an IR-camera was successfully applied to record the temperatures of numerous surface points with high speed 
and high thermal resolution. The analytical solution was written in a general form to cover most of the measuring situations of practical interest.

\section{REFERENCES}

[1] ROETZEL (W.) and PRINZEN (S.). - Measurement of local heat transfer coefficients using temperature oscillations. Experimental Heat Transfer, Fluid Mechanics and Thermodynamics, Elsevier, New York-Amsterdam-Tokyo 1991, p. 497-504

[2]WANDELT (M.) and ROETZEL (W.). - Estimation of local convective heat transfer coefficients using thermal wave analysis. Proc. 30th National Heat Transfer Conf., Portland, August 5-8, 1995, Vol. 10, p. 87-97

[3] POHLHAUSEN (E.) - Der Wărmeaustausch zwischen festen Körpem und Flüssigkeiten mit kleiner Reibung und kleiner Wămeleitung. Z. Angew. Math. Mech., No. 1, 1921, p. 115-121

[4] VDI Heat Atlas, VDI-Verlag GmbH, Düsseldorf, 1993, p. Db16-Db28

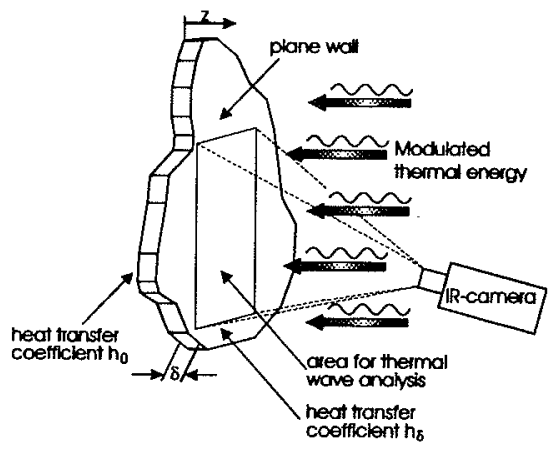

Fig. 1 : Measurement principle

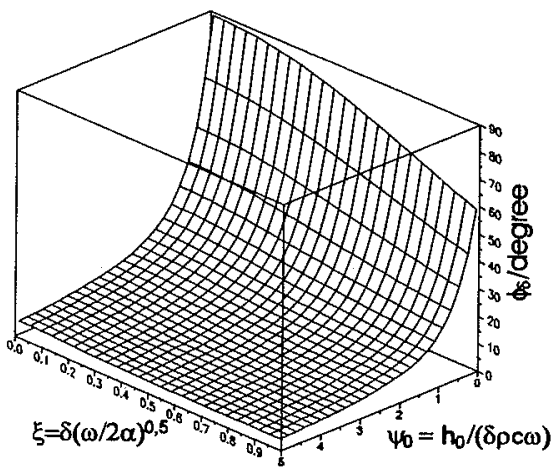

Fig. 3 : Phase $\phi_{8}$ calculated with a ratio $r=1$

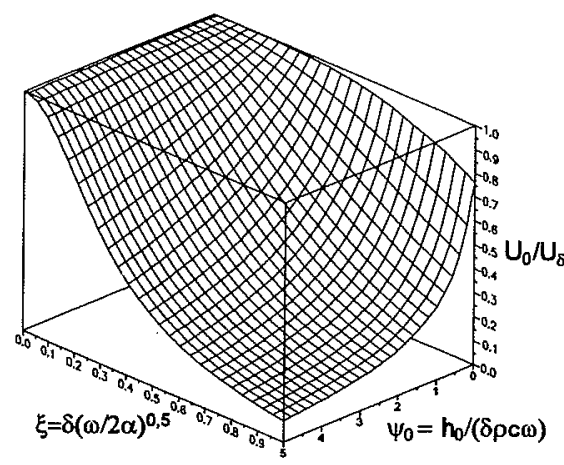

Fig. 2 : Ratio $U_{0} / U_{8}$ calculated with $r=1$

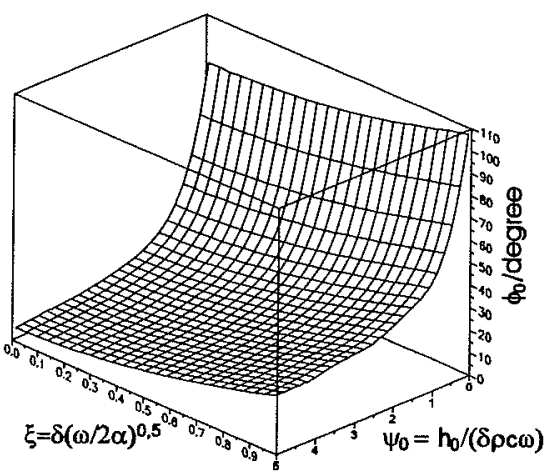

Fig. 4 : Phase $\phi_{0}$ calculated with a ratio $r=1$ 


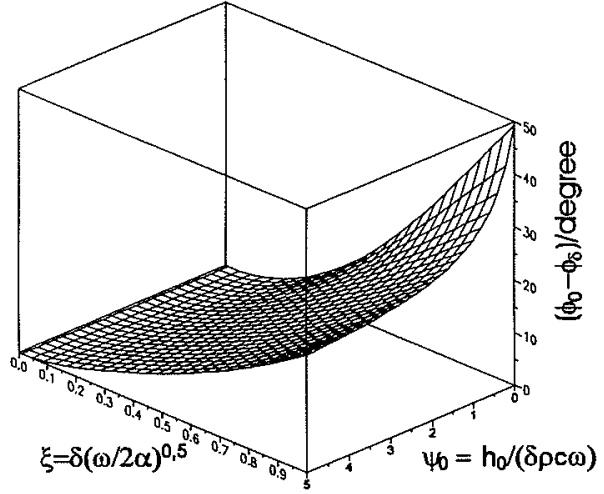

Fig. 5 : Phase lag calculated with a ratio $r=1$

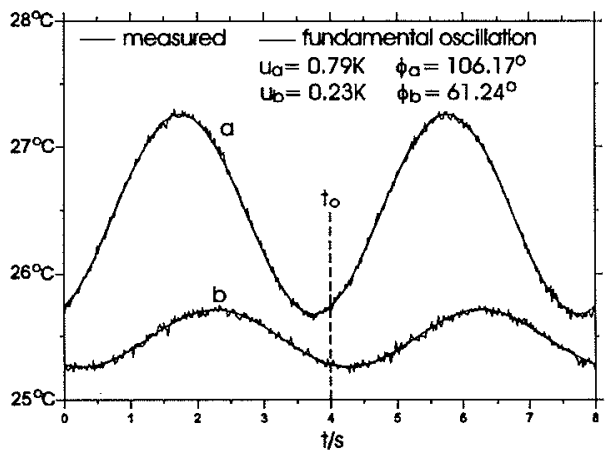

Fig. 6 : Typical temperature plots of two pixels

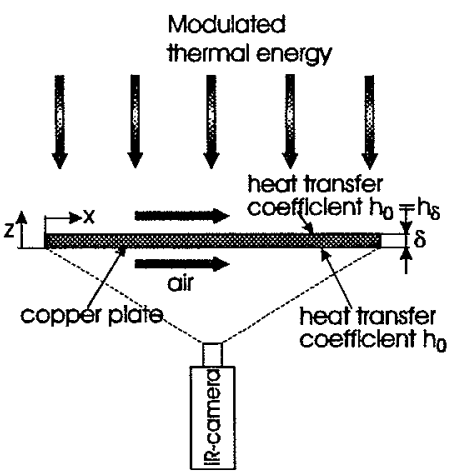

Fig. 7 : Schematic drawing of the experimental setup
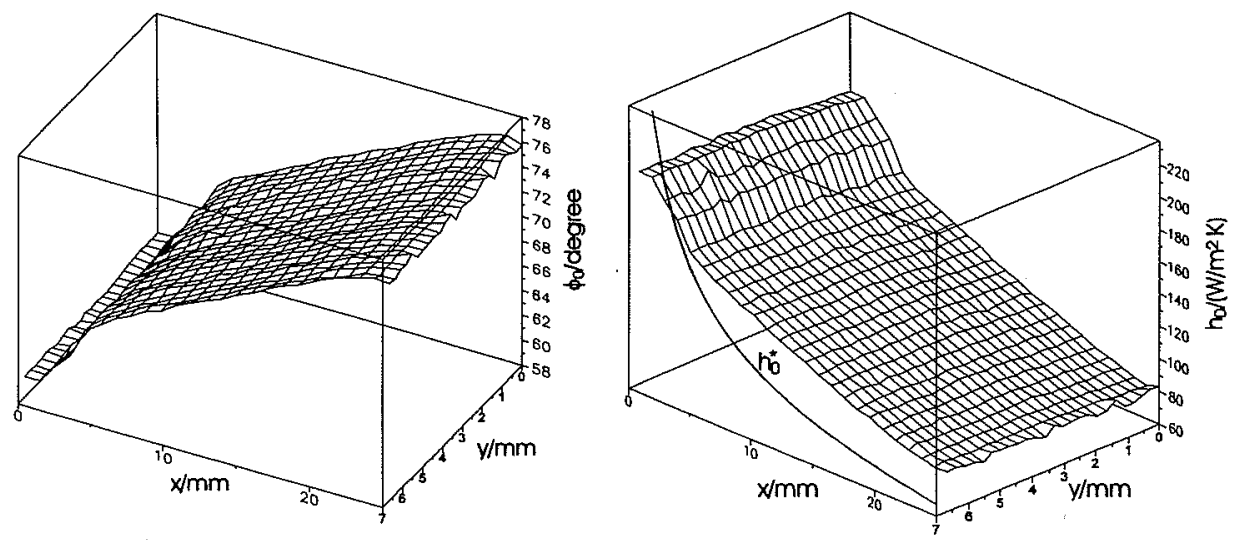

Fig. 8 : Measured phases $\phi_{0}$ and evaluated distribution of the heat transfer coefficient $h_{0}$ for a flow velocity of $30 \mathrm{~m} / \mathrm{s}$ 